\section{Corneoscleroplasty with maintenance of the angle in two cases of extensive corneoscleral disease}

REINHARD O.W. BURK, ANTONIA M. JOUSSEN
R.O.W. Burk

A.M. Joussen

Department of

Ophthalmology

University of Heidelberg

Heidelberg, Germany

A.M. Joussen, MD

Department of

Ophthalmology

RWTH Aachen

Pauwelsstrasse 30

D-52057 Aachen, Germany

Tel: +492418088 191

Fax: +49 2418888408

e-mail: JoussenA@aol.com

Presented in part at the 99th meeting of the German Ophthalmological Society, Berlin 1998

Received: 8 February 1999 Accepted in revised form: 19 November 1999

\begin{abstract}
Purpose We report a 2 year follow-up in two patients after corneoscleroplasty. Method Using lamellar corneoscleral dissection to maintain the drainage angle and its function, we performed a $14 \mathrm{~mm}$ allograft corneoscleroplasty in 2 eyes. For surgical treatment in both cases a $9.5 \mathrm{~mm}$ corneal button was excised from the recipient after peritomy and scleral lamellar preparation up to $14 \mathrm{~mm}$. A $14 \mathrm{~mm}$ donor button was inserted and held in place with multiple Prolene sutures. One eye presented with a large perforating corneal ulcer after herpetic keratitis in a patient with recurrent rheumatoid uveitis associated with rubeosis iridis. The second eye had had a penetrating keratoplasty for keratoconus 30 years previously and presented with decompensating keratoglobus. Immune suppression was performed with systemic cyclosporin $A$ and additional steroids when required.

Results Both patients had a clear graft at the last follow-up visit and visual acuity was improved to a best corrected visual acuity of 0.6. Intraocular pressure in the keratoglobus eye was maintained at $6 \mathrm{mmHg}$ without treatment, whereas the second case required continuing treatment with systemic acetazolamide because of neovascular glaucoma. The anterior chamber angles remained open in both patients. Contact lenses were helpful in the prevention of epithelial irregularities and defects. Both patients had an episode of immunological graft reaction which was reversed by immunosuppressive treatment.

Phacoemulsification with intraocular lens implantation, which was performed 2 years after transplantation in the keratoglobus eye, did not affect the graft clarity or cause rejection episodes.

Conclusion Our results using corneoscleroplasty have been encouraging in severe destructive corneal disease.
\end{abstract}

Key words Cornea, Diffuse destructive disease, Grafting, Immune suppression, Sclera

Diffuse destructive disease of the anterior segment frequently requires reconstructive keratoplasty to maintain the integrity of the eye. The large corneal grafts required in these cases are associated with a high incidence of graft rejection and glaucoma. ${ }^{1}$ Using a large graft with the attaching scleral structures means losing the immunological privilege of grafts transplanted into the clear cornea. ${ }^{2,3}$ The introduction of new immune suppressive agents such as cyclosporin A has allowed lower doses of steroids to be used, which in turn has reduced the incidence of serious systemic side effects of immune suppression. Long-term treatment is now possible. This helps to reduce the incidence of immunological graft reactions.

If the integrity of the trabecular meshwork, the drainage angle and the canal of Schlemm can be preserved, the incidence of secondary glaucoma can be reduced.

We present two cases of $360^{\circ}$ corneoscleroplasty, combining a lamellar peripheral scleral preparation with a central penetrating keratectomy. The peripheral lamellar scleral dissection avoided the trabecular meshwork and the angle in order to preserve its function. The patients were followed up for 26 and 38 months respectively.

\section{Patients and methods}

Two patients with different corneal diseases who suffered from diffuse corneal destruction and thinning received a corneoscleroplasty to provide integrity of the globe. In both patients corneal thinning and destruction of at least 2 clock-hours extending beyond the corneosclera] limbus required large grafts. In both patients conservative treatment had failed to cure the disease. Visual acuity was lower than $1 / 25$ when surgery was performed.

One of the patients suffered from recurrent anterior uveitis due to rheumatoid disease and appeared with a central corneal perforation 

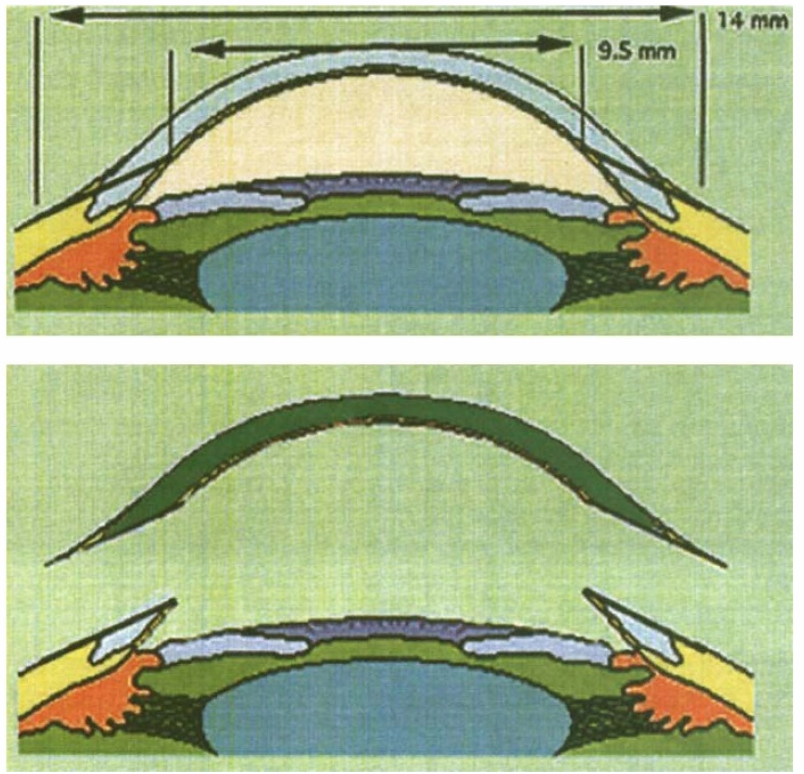

Fig. 1. The recipient graft bed was prepared as described in the text. After peritomy peripheral edges were demarcated using a $14 \mathrm{~mm}$ trephine. A central button $9.5 \mathrm{~mm}$ in diameter was excised in full thickness and the sclera was prepared circularly from a diameter of $14 \mathrm{~mm}$ towards the $9.5 \mathrm{~mm}$ demarcation line. The periphery of the graft is prepared accordingly and then the graft fitted into the recipient bed.

after herpetic keratitis. The second case was a patient with keratoconus who had developed an acute keratoglobus 32 years after a central penetrating keratoplasty.

Surgery was performed as follows in both patients: A $14 \mathrm{~mm}$ corneoscleral disc was used for each case. This was prepared using a $14 \mathrm{~mm}$ trephine. In one case the donor eye was fresh; in the other a corneoscleral disc was preserved in a short-term storage medium. The peripheral areas of the graft were prepared in a lamellar technique resulting in a split-thickness graft rim, which decreases in thickness from the peripheral cornea towards the outer (scleral) edge of the graft.

The recipient eye was prepared with a $360^{\circ}$ peritomy. Then the peripheral edges were demarcated with a 14 $\mathrm{mm}$ trephine. Sodium hyaluronate (Healon) was injected through a paracentesis to maintain the anterior chamber before excision of the corneal button and to avoid damage to the ciliary body. After marking of the central cornea with a $9.5 \mathrm{~mm}$ trephine, the sclera was prepared circularly from a diameter of $14 \mathrm{~mm}$ towards the $9.5 \mathrm{~mm}$ demarcation line. Here the anterior chamber was entered and the central $9.5 \mathrm{~mm}$ corneal button excised in full thickness (Fig. 1). Great care was taken not to affect any structures of the iridocorneal angle while preparing the graft bed.

The donor tissue was then placed over the prepared recipient sclera. The graft was first fixated with four 9-0 Prolene sutures in all quadrants. It was then sutured by up to 32 Prolene sutures from the graft rim through the recipient sclera. Viscoelastic was removed from the anterior chamber and exchanged with Balanced Salt Solution (BSS). Watertight wound closure was proven by injecting BSS into the anterior chamber and a subsequent
Seidel's test. The sutures were then tied and rotated into the outer stroma. Finally the conjunctiva was closed and attached to the graft.

\section{Case reports}

Case 1 (Fig. 2)

A 49-year-old woman with keratoconus, who had had a penetrating keratoplasty due to irregular astigmatism 32 years ago, first presented to our clinic with decreasing visual acuity in October 1994. In April 1995 the best corrected visual acuity (BCVA) had decreased to 20/400 in the affected eye, which now showed a decompensating keratoglobus with hydrops. The whole cornea was opacified and a thinning of the stroma affected the central cornea and periphery. Because the thinning reached limbal areas, a corneoscleroplasty was performed using the technique described above.

Although there was adequate wound healing, the central part of the graft showed a persistent epithelial defect, possibly due to an immunological graft reaction of the epithelium, or failure of the transplanted limbal stem cells. Epithelial defect and stromal oedema responded well to topical steroids. Systemic steroids and cyclosporin A were used to provide immune suppression. Treatment was started orally with $100 \mathrm{mg}$ prednisolone daily combined with $2 \times 200 \mathrm{mg}$ cyclosporin A with reduction of steroid dose thereafter. The blood cyclosporin level was kept at $0.25 \mathrm{mg} / \mathrm{dl}$, which is within the therapeutic range $(0.14-0.3 \mathrm{mg} / \mathrm{dl})$.

On admission, the BCVA was 20/200, but increased to $20 / 80$ (pinhole) after completion of epithelial healing. Without local ocular hypertensive treatment the intraocular pressure was stable between 10 and $15 \mathrm{mmHg}$ (appl.).

The patient had an allograft rejection episode 5 months after transplantation with endothelial deposits and epithelial irregularities. Systemic immunosuppressive treatment was increased to $100 \mathrm{mg} /$ day prednisolone i.v. (1.5 $\mathrm{mg} / \mathrm{kg}$ body weight) whereas the cyclosporin A level was kept at $0.25 \mathrm{mg} / \mathrm{dl}$. Topical steroid treatment was also increased (Ultracortenol ointment (triamcinolone) t.d.s.) and epithelial irregularities were covered by a bandage contact lens. With this therapy epithelial healing was achieved. The intraocular pressure was maintained at $8 \mathrm{mmHg}$ (appl.).

Immunosuppressive treatment with cyclosporin A was continued under control of blood and retention parameters for the following 24 months. Systemic corticosteroids were stopped to avoid systemic side effects.

An advanced cataract developed in February 1997 and visual acuity decreased to hand movements. Therefore phacoemulsification and posterior chamber lens (Storz, PMMA, 14 mm, +31 dpt.) implantation was performed (power was estimated by comparison with the other eye). Postoperatively the corneal graft remained clear. The visual acuity at 2 months after surgery was $20 / 30$ with a $-1.75 /+1.25 \times 95^{\circ}$. 


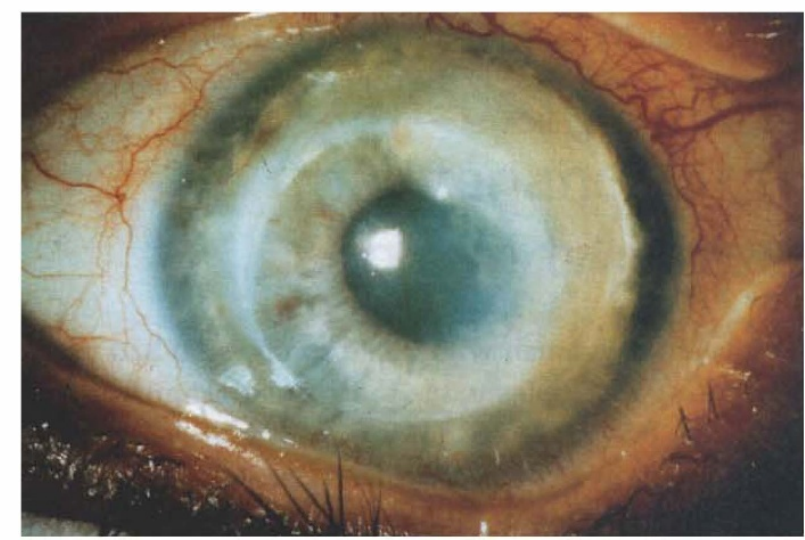

(a)

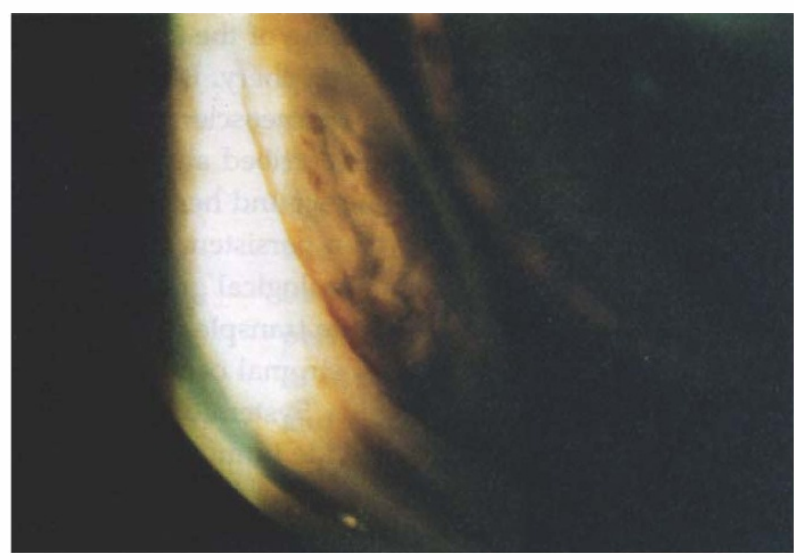

(c)

\section{Case 2 (Fig. 3)}

A 43-year-old woman with rheumatoid arthritis developed a central perforating corneal ulcer with iris prolapse. She showed significant rubeosis of the iris and reported a history of recurrent anterior uveitis and for the last 10 years recurrent herpetic keratitis. Her visual acuity had decreased to hand movements and light perception 1 year previously as she developed a cataracta complicata. When she first presented to our clinic the anterior chamber was flat and there was no view of the posterior segment. Seidel's test was positive for 8 days. On the 19 April 1996 a corneoscleroplasty was performed as described above. Extracapsular cataract extraction was performed. Because of the dense sclerotic posterior capsule a posterior capsulectomy and anterior vitrectomy had to be done in the same session. An intraocular lens was not implanted at the time of transplantation due to rubeosis iridis. Funduscopy showed a vertically elongated excavation of the optic disc cup with a cup:disc ratio of 0.7 . To avoid anterior chamber bleeding, rubeotic iris vessels were coagulated intraoperatively with a diode laser. As described in case 1 're-epithelialisation' was relatively slow but was achieved with the help of a bandage contact lens. Visual acuity increased to $20 / 80$ using a correction of $+10 \mathrm{D}$ in August 1996.

A few days later the patient lost visual acuity again due to an atypical immunological transplant reaction with signs of iritis and a corneal stromal oedema and

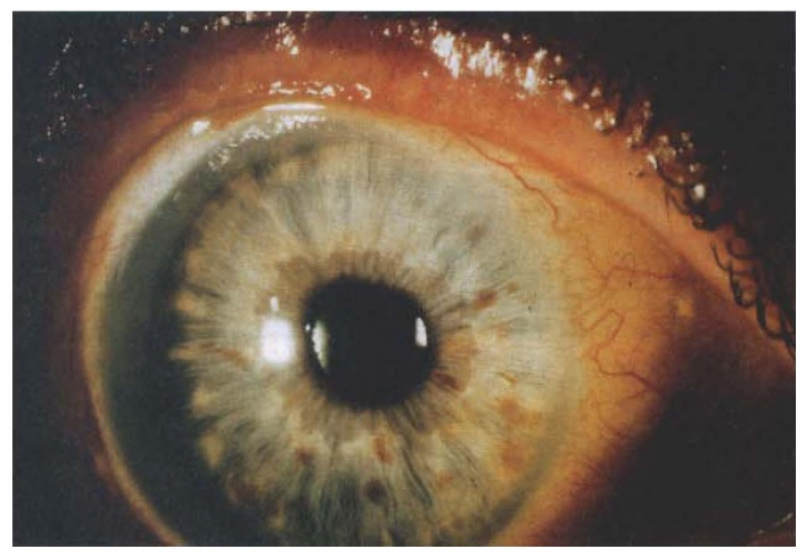

(b)

Fig. 2. Patient 1. (a) Decompensated keratoconus 32 years after penetrating keratoplasty. (b) Thirty-six months after corneoscleroplasty. (c) Regular anterior chamber angle after corneoscleroplasty.

recurrent epithelial defect. Paracentrally there was a stromal delle. Treatment with topical and systemic cyclosporin A (200 mg/day; $0.04 \%$ eye drops q.i.d.) was successful in controlling the destructive process. A soft contact lens was used because of the persistent corneal epithelial defect. There was stromal thinning to one-third of the stromal thickness, which covered the whole central area of the cornea. The stromal thinning was resistant to therapy, but there was no sign of progression at any time, nor signs of stromal opacification.

The immunological transplant reaction caused raised intraocular pressure, which responded well to Diamox (acetazolamide) orally. As pressure measurements were difficult due to thinning and softness of the transplant, treatment with acetazolamide in a daily dose of $250 \mathrm{mg}$ was continued for 3 weeks.

With the contact lens visual acuity remained stable at 20/50 until January 1998. At the last visit local treatment was cyclosporin A drops and Dexasine drops (dexamethasone eye drops without preservatives), each two times daily, and Floxal (ofloxacine) drops once a day. At the last follow-up visit cyclosporin treatment was already reduced below the therapeutic blood level.

\section{Results}

Two patients with diffuse corneoscleral disease were followed up for 26 and 38 months respectively after combined lamellar and perforating corneoscleroplasty. Both patients had an episode of immunological graft reaction which responded well to topical and systemic immunosuppressive treatment. Both transplants have remained clear during the observation period to date. A re-graft or a central perforating keratoplasty for visual rehabilitation was not necessary in either of the patients. In both patients systemic immunosuppression with cyclosporin A was continued, extending the observation period. 


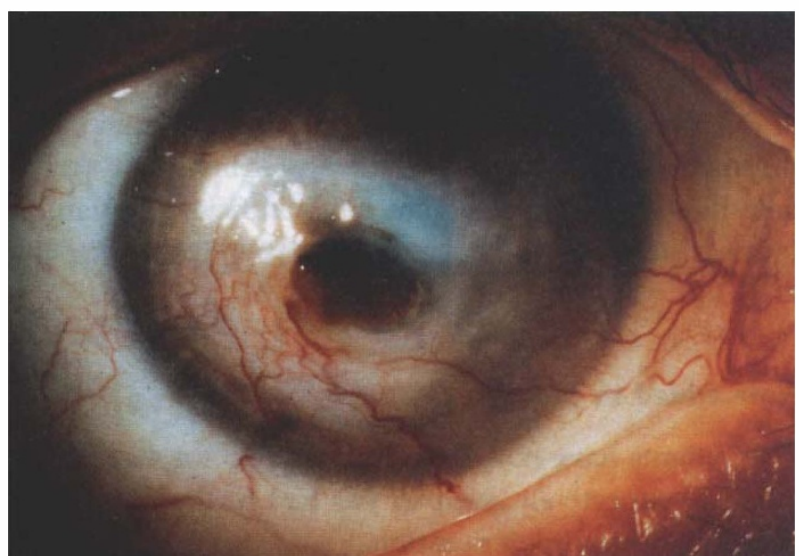

(a)

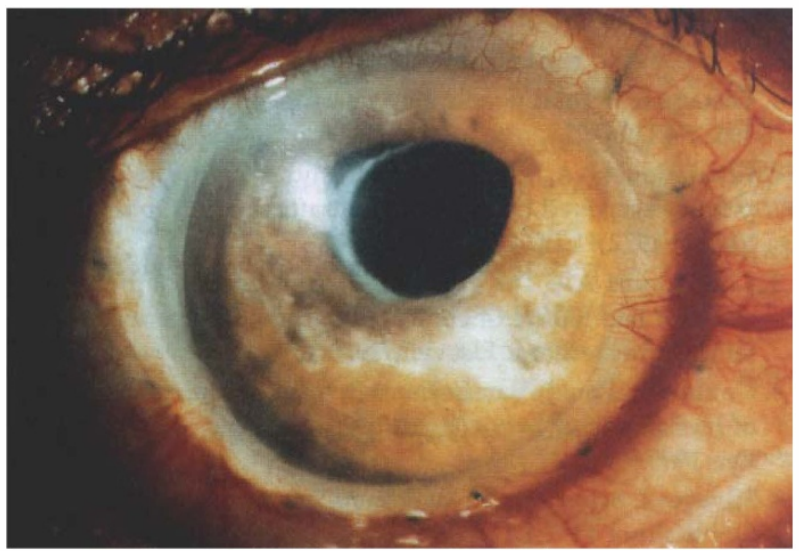

(c)

Although there was a significant overall thinning of the graft stroma in one of the patients, no localised destruction requiring therapy occurred. In both cases either soft bandage contact lenses or a soft lens for correction of aphakia was required to prevent persistent central epithelial defects, especially since the central epithelium was fragile in both patients.

Due to progression of a 'pre-existing' nuclear cataract, one patient required cataract surgery, which was performed without damage to the graft. At the last follow-up visit visual acuity had improved in both patients compared with the pre-operative level.

For the first 5 months the intraocular pressure remained within normal limits after surgery. In one of the patients intraocular pressure rose after enhanced topical steroid treatment for an episode of immunological transplant reaction and required treatment with systemic acetazolamide. Use of long-term topical antiglaucomatous treatment was avoided in order to prevent epithelial damage. The anterior chamber angle remained open in both patients.

\section{Discussion}

If corneal thinning extends to the limbal areas and reasonably quick visual rehabilitation is necessary, a large corneoscleroplasty is an option. One of the major problems with larger corneal grafts is the high risk of graft rejection. ${ }^{4}$.If the corneal graft extends to the limbal

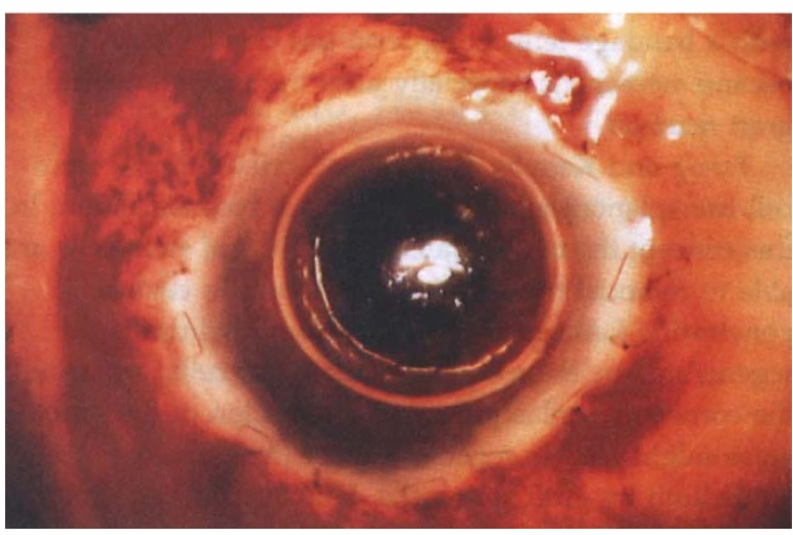

(b)

Fig. 3. Patient 2. (a) Perforated corneal ulcer. (b) Intraoperative status after corneoscleroplasty. (c) Twenty-seven months after corneoscleroplasty.

area, the so-called immunological privilege of corneal allografts, which are sutured into an avascular recipient cornea as described by Khoudadoust, is lost. ${ }^{2,3}$ The corneoscleral graft, in contrast to the central corneal graft, attaches the limbal vasculature of the recipient to the antigenic donor tissue.

Despite these well-known problems a large graft is sometimes necessary due to peripheral corneal involvement. Whereas in many cases only a small eccentric graft is required, ${ }^{5}$ extensive corneal thinning sometimes requires excision of the whole diseased corneal and limbal tissue. ${ }^{6}$

Sundmacher and Reinhard ${ }^{7,8}$ described the successful use of cyclosporin A in high-risk keratoplasties.

Although both our patients received immunosuppressive treatment with cyclosporin A in therapeutic dosage, both developed an atypical immunological graft reaction when the additional steroid treatment was reduced for the first time in order to continue immunosuppression with cyclosporin alone. After elevation of steroid treatment the immunological process was stopped in both cases and did not recur, even though steroid treatment was slowly decreased. This might be due to an activation of the immune system by the underlying disease.

To avoid the potential risks of immunosuppression, Reinhard and co-workers ${ }^{9}$ suggested performing a lamellar corneoscleroplasty with thermoplasty for peripheral corneal ectasia. Both our patients required full-thickness transplantation of the whole cornea because of the extent of the destructive process in both depth and in size. We therefore accepted the risk of immune suppression in order to increase the graft survival. Blood cell counts and creatinine levels were examined on a regular basis.

Although graft failure was prevented in both patients by immune suppression, both developed recurrent central epithelial irregularities. Epithelial healing was delayed in both patients after surgery, but was achieved 
with a bandage lens. Long-term use of a contact lens became necessary, especially after the immunological graft reaction.

Tseng and Kenyon ${ }^{10-12}$ have described limbal stem cell transplantation for ocular surface reconstruction. In this concept the newly transplanted limbal stem cells are able to remodel the corneal epithelium. In central penetrating keratoplasties the graft epithelium is regenerated by the recipient limbal stem cells. Regarding the corneoscleroplasty the transplantation of the limbal areas might lead to regeneration of the corneal epithelium by graft stem cells. However, the prolonged epithelial irregularities in both patients are probably due to impaired stem cell function. In our patients we did not observe progressive peripheral crystalline deposits as seen by Slowik et al. ${ }^{13}$ or any signs of loss of graft clearity. The central graft remained clear after phacoemulsification for complicated cataract and implantation of a posterior chamber lens in one of the patients.

Probably due to the transplantation of the whole cornea with the adjacent scleral tissue, the patient with previous keratoglobus did not develop recurrent disease. In contrast there was a central thinning of the graft in the rheumatoid patient. This might indicate that, in keratoglobus or keratoconus, the dysfunction of the fibroblasts is limited to the cornea, whereas in rheumatoid disease a systemic process is involved. Therefore corneal thinning can recur, even if the whole affected cornea is excised. In this case the immune suppression may help to prevent further corneal involvement.

Besides prevention of immunological graft rejections, another of the major risk factors for graft failure is postoperative secondary glaucoma or hypotony. Several authors have attempted to prevent elevation of intraocular pressure with measures such as intraoperative subtotal cyclodialysis or trabeculectomy. ${ }^{14}$ Cobo and co-workers ${ }^{15}$ used angle support sutures combined with a lamellar dissection and observed gonioscopically open angles in all patients. The authors describe elevated intraocular pressure in one patient upon topical steroid treatment. In one of our cases (the keratoglobus patient) there was normal intraocular pressure. The other case had pre-operative rubeosis iridis and did have raised intraocular pressure, especially after the immunological graft rejection. Acetazolamide was used to minimise the number of local treatments. This was successful in controlling the intraocular pressure.

To avoid the necessity of angle-opening sutures we tried to prevent damage to the structures of the corneoscleral angle during lamellar preparation. In neither case was there an angle closure or other mechanical problem with aqueous outflow.
In conclusion, a combined lamellar and perforating large corneoscleroplasty is able to preserve the integrity of the globe and the anterior segment. Prevention of destruction of the angle and trabecular meshwork can help to avoid secondary glaucoma. Sufficient systemic immunosuppression is necessary to keep the graft clear.

\section{Note added in proof}

The patients have now been followed up for 40 and 50 months, respectively. Neither has had further rejection periods or visual deterioration.

\section{References}

1. Barraquer J. Total penetrating keratoplasty. Proc R Soc Med 1961;54:1116-21.

2. Khodadoust AA, Silverstein AM. Studies on the nature of the privilege enjoyed by corneal allografts. Invest Ophthalmol Vis Sci 1972;11:137-48.

3. Khodadoust AA. The allograft rejection reaction: the leading cause of late failure of clinical allografts. In: Corneal graft failure. Amsterdam: Elsevier, 1973:151-67.

4. Goulmy E, Pool J, Van Lochem E, Völker-Dieben H. The role of human minor histocompatibility antigens in graft failure. Eye 1995;9:180-4.

5. Süveges I. Sclerokeratoplasty in recurrent pterygium. Germ J Ophthalmol 1992;1:114-16.

6. Coster DJ. An alternative approach to corneoscleral repair [editorial]. Br J Ophthalmol 1993;77:325.

7. Reinhard T, Sundmacher R, Heering P. Systemic cyclosporin A in high-risk keratoplasties. Graefes Arch Clin Exp Ophthalmol 1996;234:S115-21.

8. Sundmacher R, Reinhard T. Central corneolimbal transplantation under systemic cyclosporin A cover for severe limbal stem cell insufficiency. Graefes Arch Clin Exp Ophthalmol 1996;234:S122-5.

9. Reinhard T, Sundmacher R. Lamelläre Horse-Shoe Sklerokeratoplastik und Thermoplastik bei Keratokonus mit peripherer Keratektasie. Klin Monatsbl Augenheilkd 1994;205:305-8.

10. Kenyon KR, Tseng SC. Limbal autograft transplantation for ocular surface disorders. Ophthalmology 1989;96:709-23.

11. Tseng SC. Concept and application of limbal stem cells. Eye 1989;3:141-57.

12. Tseng SC, Tsai RJ. Limbal stem cell transplantation for ocular surface reconstruction: a review. Fortschr Ophthalmol 1991;88:236-42.

13. Slowik C, Somodi S, Richter A, Guthoff R. Konfokale in vivo Mikroskopie mit $15 \mathrm{~mm}$ Sklerokorneoplastik à chaud bei nekrotisierender Keratitis. Klin Monatsbl Augenheilkd 1996;208:246-50.

14. Taylor DM, Stern AL. Reconstructive keratoplasty in the management of conditions healing to corneal destruction. Ophthalmology 1980;87:892-9.

15. Cobo M, Ortiz JR, Safran SG. Sclerokeratoplasty with maintenance of the angle. Am J Ophthalmol 1992;113:533-7. 